\title{
Recommendation for use of diphtheria and tetanus toxoids and acellular pertussis, inactivated poliovirus, Haemophilus influenzae type b conjugate, and hepatitis B vaccine in infants
}

Hye-Kyung Cho, MD, PhD ${ }^{1}$, Su Eun Park, MD, PhD², Yae-Jean Kim, MD, PhD ${ }^{3}$, Dae Sun Jo, MD, PhD ${ }^{4}$, Yun-Kyung Kim, MD, $\mathrm{PhD}^{5}$, Byung-Wook Eun, MD, PhD ${ }^{6}$, Taek-Jin Lee, MD, PhD' , Jina Lee, MD, PhD ${ }^{8}$, Hyunju Lee, MD, PhD ${ }^{9}$, Ki Hwan Kim, MD, $\mathrm{PhD}^{10}, *$, Eun Young Cho, MD, PhD ${ }^{11}$, Jong Gyun Ahn, MD, PhD ${ }^{12}$, Eun Hwa Choi, MD, PhD ${ }^{9}$; The Committee on Infectious Diseases of the Korean Pediatric Society

${ }^{1}$ Department of Pediatrics, Gachon University Gil Medical Center, Gachon University College of Medicine, Incheon, Korea; ${ }^{2}$ Department of Pediatrics, School of Medicine, Pusan National University, Pusan, Korea; ${ }^{3}$ Department of Pediatrics, Sungkyunkwan University School of Medicine, Suwon, Korea; ${ }^{4}$ Department of Pediatrics, Jeonbuk National University Medical School, Jeonju, Korea; ${ }^{5}$ Department of Pediatrics, Korea University College of Medicine, Seoul, Korea; ${ }^{6}$ Department of Pediatrics, School of Medicine, Eulji University, Daejeon, Korea; ${ }^{7}$ Department of Pediatrics, CHA University School of Medicine, Pocheon, Korea; ${ }^{8}$ Department of Pediatrics, University of Ulsan College of Medicine, Ulsan, Korea; ${ }^{9}$ Department of Pediatrics, Seoul National University College of Medicine, Seoul, Korea; ${ }^{10}$ Department of Pediatrics, College of Medicine, The Catholic University of Korea, Seoul, Korea; ${ }^{11}$ Department of Pediatrics, Chungnam National University School of Medicine, Daejeon, Korea; ${ }^{12}$ Department of Pediatrics, Severance Children's Hospital, Yonsei University College of Medicine, Seoul, Korea

In April 2020, the Ministry of Food and Drug Safety licensed a hexavalent combined diphtheria and tetanus toxoids and acellular pertussis (DTaP), inactivated poliovirus (IPV), Haemophilus influenzae type $\mathrm{b}(\mathrm{Hib})$ conjugated to tetanus protein, and hepatitis B (HepB) (recombinant DNA) vaccine, DTaPIPV-Hib-HepB (Hexaxim, Sanofi Pasteur), for use as a 3-dose primary series in infants aged 2,4 , and 6 months. The DTaP-IPVHib-HepB vaccine is highly immunogenic and safe and provides a long-term immune response based on studies performed in a variety of settings in many countries, including Korea. This report summarizes the Committee on Infectious Diseases of the Korean Pediatric Society guidelines for the use of this newly introduced hexavalent combination vaccine.

Key words: Vaccines, Combined, Diphtheria-tetanus-acellular pertussis-inactivate, Infant

\section{Key message}

- Diphtheria and tetanus toxoids and acellular pertussis-inactivated poliovirus-Haemophilus influenzae type b-hepatitis B (DTaP-IPV-Hib-HepB) was licensed in Korea in April 2020.

- DTaP-IPV-Hib-HepB is indicated as a 3-dose primary series for infants aged 2, 4, and 6 months who received the standalone HepB vaccine at birth.

- Infants born to HepB surface antigen-positive mothers are currently recommended to be immunized with HepB immunoglobulin at birth and then monovalent $\mathrm{HepB}$ vaccine at 0,1 , and 6 months.

\section{Introduction}

Combination vaccines, compared with individually administered components, decrease the number of injections, amount of pain, and cumulative exposure to preservatives and stabilizers that can contribute to adverse events. ${ }^{1)}$ Moreover, combination products have simplified immunization delivery, improving vaccine coverage and vaccination timeliness with additional cost-saving effects of administration, handling, and storage..$^{2-4)}$ In Korea, tetravalent and pentavalent vaccines were introduced in 2009 and 2017, respectively, based on the results of clinical trials in Korean infants. ${ }^{5-7)}$ In April 2020, the Ministry of Food and Drug Safety licensed a hexavalent combined diphtheria and tetanus toxoids and acellular pertussis (DTaP), inactivated poliovirus (IPV), Haemophilus influenzae type b (Hib) conjugated to tetanus protein, and hepatitis B (HepB) (recombinant DNA) vaccine, DTaP-IPV-Hib-HepB (Hexaxim; Sanofi Pasteur, Lyon, France), for use as a 3-dose primary series in infants aged 2, 4 , and 6 months. ${ }^{8)}$ The DTaP-IPV-Hib-HepB vaccine is not currently included in the national immunization program.

There are currently 3 hexavalent vaccine preparations approved for use worldwide: Infanrix-hexa (GSK, Rixensart, Belgium); Hexyon/Hexacima/Hexaxim (all Sanofi Pasteur); and Vaxelis (MSP Vaccine Co., Swiftwater, PA, USA). ${ }^{9)}$ Their composition and formulation differ slightly, but all have shown good immunogenicity and safety profiles. ${ }^{9-11)}$ Hexyon and Hexacima are the same products as Hexaxim, with the only differences being their brand names depending on the country of sale..$^{9}$ As of 2021, only Hexaxim has been introduced in Korea. This report

\footnotetext{
Corresponding author: Eun Hwa Choi, MD, PhD, Department of Pediatrics, Seoul National University College of Medicine, 101 Daehak-ro, Jongno-gu, Seoul 03080, Korea 凶Email: eunchoi@snu.ac.kr, https://orcid.org/0000-0002-5857-0749

*Current Affiliation: Yonsei Pediatric Clinic, Uijeongbu, Korea

Received: 21 April, 2021, Revised: 12 May, 2021, Accepted: 14 May, 2021

This is an open-access article distributed under the terms of the Creative Commons Attribution Non-Commercial License (http://creativecommons.org/licenses/by-nc/4.0/) which permits unrestricted non-commercial use, distribution, and reproduction in any medium, provided the original work is properly cited.

Copyright (c) 2021 by The Korean Pediatric Society
} 


\begin{tabular}{|c|c|c|c|c|c|c|}
\hline & & At birth & 1 month & 2 months & 4 months & 6 months \\
\hline \multirow{3}{*}{$\begin{array}{l}\text { When vaccinated with } \\
\text { tetravalent or pentavalent } \\
\text { vaccine }\end{array}$} & Monovalent HepB & $\sqrt{\text { a) }}$ & $\sqrt{ }$ & & & $\sqrt{ }$ \\
\hline & $\mathrm{DTaP} / \mathrm{IPV}+\mathrm{Hib}$ & & & $\sqrt{ } \sqrt{ }$ & $\sqrt{ } \sqrt{ }$ & $\sqrt{ } \sqrt{ }$ \\
\hline & DTaP-IPV/Hib & & & $\sqrt{ }$ & $\sqrt{ }$ & $\sqrt{ }$ \\
\hline \multirow{2}{*}{$\begin{array}{l}\text { When vaccinated with } \\
\text { hexavalent vaccine }\end{array}$} & Monovalent HepB & $\sqrt{ }$ & & & & \\
\hline & DTaP-IPV-Hib-HepB & & & $\sqrt{ }$ & $\sqrt{ }$ & $\sqrt{ }$ \\
\hline
\end{tabular}

Graphic abstract

summarizes the Committee on Infectious Diseases of the Korean Pediatric Society guidelines for the use of a newly introduced hexavalent combination vaccine.

\section{Composition of the hexavalent vaccine}

The DTaP-IPV-Hib-HepB vaccine is based on the wellestablished pentavalent DTaP-IPV/Hib vaccine (Pentaxim; Sanofi Pasteur) ${ }^{12)}$ with the addition of $10 \mu \mathrm{g}$ of Hansenula polymorphaderived HepB surface antigen (HBsAg). Each dose contains the same amount of diphtheria and tetanus toxoids and pertussis antigens (inactivated pertussis toxin [PT] and filamentous hemag. glutinin [FHA]) and the same amount of inactivated poliovirus antigens as DTaP-IPV/Hib (Table 1). The Hib component (Hib capsular polysaccharide polyribosyl-ribotol-phosphate [PRP] coupled to tetanus toxoid protein) is the same as that in DTaP-IPV/ Hib but in an increased amount. It features a preservative-free fully liquid formulation that requires no reconstitution. Since
Hexaxim was first licensed in Europe in 2013, it has been licensed in more than 100 countries worldwide with more than 30 million doses distributed in 70 countries, and it is pre-qualified by the World Health Organization. ${ }^{13,14)}$

\section{Immunogenicity}

The immunogenicity of the DTaP-IPV-Hib-HepB vaccine has been evaluated in a variety of settings in many countries worldwide. It is highly immunogenic compared to licensed comparators. ${ }^{15-20)}$

A phase III noninferiority study evaluated the immunogenicity of DTaP-IPV-Hib-HepB vaccine in $>300$ Korean infants. ${ }^{19)}$ The immunologic responses were compared between the 2 groups: infants vaccinated with $\mathrm{HepB}$ vaccine alone at birth and hexavalent vaccine at 2, 4, and 6 months of age (group 1); and infants vaccinated with a standalone $\mathrm{HepB}$ vaccine at birth and at 1 and 6 months of age and a pentavalent vaccine (DTaP-IPV/Hib)

Table 1. Composition of the newly introduced DTaP-IPV-Hib-HepB vaccine (Hexaxim, Sanofi Pasteur, Lyon, France) vs. DTaP-IPV/Hib (Pentaxim, Sanofi Pasteur) $^{7,33)}$

\begin{tabular}{|c|c|c|c|}
\hline \multirow[b]{2}{*}{ Ingredient } & \multicolumn{2}{|c|}{ Concentration per $0.5-\mathrm{mL}$ dose } & \multirow[b]{2}{*}{ Function } \\
\hline & $\begin{array}{l}\text { DTaP-IPV-Hib-HepB } \\
\text { (Hexaxim) }\end{array}$ & $\begin{array}{l}\text { DTaP-IPV/Hib } \\
\text { (Pentaxim) }\end{array}$ & \\
\hline Diphtheria toxoid & $30 \operatorname{Lf}\left(\geq 20 \| U^{\mathrm{a})}\right)$ & $30 \mathrm{Lf}\left(\geq 20 I^{\mathrm{a})}\right)$ & Active ingredient \\
\hline Tetanus toxoid & $10 \mathrm{Lf}\left(\geq 40 \mid U^{\mathrm{a})}\right)$ & $10 \mathrm{Lf}\left(\geq 40 \mid U^{\mathrm{a})}\right)$ & Active ingredient \\
\hline Bordetella pertussis antigens & & & Active ingredient \\
\hline Pertussis toxoid & $25 \mu \mathrm{g}$ & $25 \mu \mathrm{g}$ & \\
\hline Filamentous hemagglutinin & $25 \mu \mathrm{g}$ & $25 \mu \mathrm{g}$ & \\
\hline Polio virus (Mahoney) type $1^{\text {b) }}$ & $40 \mathrm{D}$-antigen units & 32 D-antigen units & Active ingredient \\
\hline Polio virus (MEF-1) type $2^{\text {b) }}$ & 8 D-antigen units & 8 D-antigen units & \\
\hline Polio virus (Saukett) type $3^{\text {b) }}$ & $40 \mathrm{D}$-antigen units & $32 \mathrm{D}$-antigen units & \\
\hline $\begin{array}{l}\text { Haemophilus influenza type b polysaccharide (polyribosyl-ribitol- } \\
\text { phosphate [PRP]) conjugated to tetanus protein (PRP-T) }\end{array}$ & $12 \mu \mathrm{g}$ & $10 \mu \mathrm{g}$ & Active ingredient \\
\hline Hepatitis B surface antigenc) & $10 \mu \mathrm{g}$ & None & Active ingredient \\
\hline Aluminium hydroxide, hydrated, for adsorption & $0.6 \mathrm{mg} \mathrm{Al}^{3+}$ & $0.3 \mathrm{mg} \mathrm{Al}^{3+}$ & Adjuvant \\
\hline Buffer solution & & & $\begin{array}{l}\text { Neutralization, osmolality, } \\
\text { and adjustment }\end{array}$ \\
\hline
\end{tabular}

DTaP, diphtheria and tetanus toxoids and acellular pertussis; IPV, inactivated poliovirus; Hib, Haemophilus influenzae type b; HepB, hepatitis B.

${ }^{a}$ As lower confidence limit $(P=0.95)$. ${ }^{\text {b) }}$ Produced on Vero cells. ${ }^{c}$ Produced in yeast Hansenula polymorpha cells using recombinant DNA technology 
at 2, 4, and 6 months of age (group 2). ${ }^{19)}$ For anti-HBs $(\geq 10 \mathrm{mIU} /$ $\mathrm{mL}),-\mathrm{PRP}(\geq 0.15 \mu \mathrm{g} / \mathrm{mL}),-\mathrm{D}(>0.01 \mathrm{IU} / \mathrm{mL}),-\mathrm{T}(0.1 \mathrm{IU} / \mathrm{mL})$, -polio 1, 2, and 3 ( $\geq 8$ [1/dil]), seropositive rates were $\geq 97.7 \%$ and $\geq 96.9 \%$ in groups 1 and 2, respectively. ${ }^{19)}$ For anti-PT and anti-FHA, seroconversion ( $\geq 4$-fold antibody concentration increase from prevaccination of the first dose to postvaccination of the third dose) was $\geq 91.7 \%$ and $\geq 89.3 \%$ in groups 1 and 2 , respectively. ${ }^{19)}$ The geometric mean concentrations (GMCs) and geometric mean titers of antibodies for all antigens were comparable between the 2 groups except that anti-HBs GMC was slightly higher in group 1 than in group $2(1,068 \mathrm{mIU} / \mathrm{mL}$ vs. $827 \mathrm{mIU} / \mathrm{mL}$ ), confirming the noninferiority of the hexavalent vaccine. ${ }^{19)}$

\section{Long-term immunity}

A former hexavalent vaccine, Hexavac (Sanofi Pasteur), licensed in Europe in 2000, could not guarantee long-term protective immunity against HepB; it was withdrawn in 2005. ${ }^{21)} \mathrm{It}$ was presumed that the small amount of antigen $(5 \mu \mathrm{g})$ contained in the vaccine did not provide long-term persistence of antiHBs antibodies. ${ }^{22,23)}$ In contrast, another hexavalent vaccine (Infanrix-hexa, GSK), also licensed in Europe in 2000, which contains $10 \mu \mathrm{g}$ of HBsAg, reportedly provides persistent protective immunity. Immunization with a standalone $H e p B$ vaccine at birth and a 3-dose primary series with Hexaxim, which contains $10 \mu \mathrm{g}$ of $\mathrm{HBsAg}$, at 2, 4, and 6 months of age, reportedly induces a long-lasting anti-HBs antibody response and persistent immune memory up to 9-10 years of age, comparable with the results of immunization with Infanrix-hexa. ${ }^{14)}$

\section{Safety}

The safety profile of DTaP-IPV-Hib-HepB was comparable to that of DTaP-IPV/Hib. In a clinical trial conducted in Korean infants, there were no immediate AEs. ${ }^{19)}$ Local adverse reactions such as pain, erythema, and swelling commonly occurred (48\%$62 \%$ ), comparable to those of DTaP-IPV/Hib (43\%-58\%). ${ }^{19}$ Systemic adverse reactions such as vomiting, crying, somnolence, decreased appetite, and irritability, except for fever, occurred in subjects who received DTaP-IPV-Hib-HepB (27\%-57\%), similar to those who received DTaP-IPV/Hib (25\%-49\%). Fever occurred more frequently in the DTaP-IPV-Hib-HepB group (20.1\%; 95\% confidential interval [CI], 14.0-27.5) than in the DTaP-IPV/Hib group (7.7\%; 95\% CI, 4.1-13.1); the overall differences in the incidence of fever came from after the first and second doses (8.1\% and 1.3\%, 9.4\%, and 2.6\%, respectively). ${ }^{19}$ ) A high fever $>39.5^{\circ} \mathrm{C}$ did not occur in either group. No intergroup difference was observed regarding the incidence of severe adverse events. ${ }^{19)}$

\section{Indication for use}

DTaP-IPV-Hib-HepB is licensed for use in children aged $\geq 2$ months. ${ }^{8)}$ DTaP-IPV-Hib-HepB is indicated only for use as a 3-dose series in infants who received a standalone $\mathrm{HepB}$ vaccine at birth. For infants who received the first dose of the HepB vaccine at birth, a 3-dose primary series of DTaP-IPV-Hib-HepB can be administered at 2,4 , and 6 months of age.

\section{Prevention of diphtheria, tetanus, and pertussis}

Children are recommended to receive a 3-dose primary series of DTaP at ages 2, 4, and 6 months as well as booster doses at ages 15-18 months and 4-6 years. ${ }^{7)}$ DTaP-IPV-Hib-HepB can be used for the first 3 doses of the recommended DTaP series but should not be used for the fourth or fifth dose. However, if DTaP-IPVHib-HepB is inadvertently administered for either booster dose, it need not be repeated with another DTaP-containing vaccine when spacing between the previous doses was proper. The recommended minimum age for the third dose of the DTaP-IPVHib-HepB vaccine is 24 weeks, the minimum age for completion of the HepB vaccine series. Therefore, this combination vaccine is not recommended for use in the third dose of the primary series on an accelerated schedule at 4-week intervals to prevent pertussis (Table 2).

\section{Prevention of poliomyelitis}

Children are recommended to receive 4 doses of IPV, at ages 2, 4, 6-18 months, and 4-6 years. ${ }^{24)}$ DTaP-IPV-Hib-HepB may be used for the first 3 doses of the IPV series but is not indicated for the fourth dose. However, if DTaP-IPV-Hib-HepB is inadvertently given for the fourth dose, it need not be repeated with another IPV-containing vaccine when spacing between the previous doses was proper.

\section{Prevention of invasive Hib disease}

Children are recommended to receive a primary series of a Hib conjugate vaccine and a booster dose of vaccine at 12-15

Table 2. Recommended minimum ages and intervals between doses for DTaP-IPV-Hib-HepB vaccine administration

\begin{tabular}{lcc}
\hline Dose & Minimum age & Minimum interval to next dose \\
\hline First dose & 6 Weeks & 4 Weeks \\
Second dose & 10 Weeks & 4 Weeks \\
Third dose & 24 Weeks $^{\text {a) }}$ & -
\end{tabular}

DTaP, diphtheria and tetanus toxoids and acellular pertussis; IPV, inactivated poliovirus; Hib, Haemophilus influenzae type b; HepB, hepatitis B.

a) If the third vaccination is given before 24 weeks of age, an additional dose of monovalent hepatitis $B$ vaccine should be administered at age $\geq 24$ weeks to complete the hepatitis B series (if vaccine components other than HepB were properly spaced). 
months of age. ${ }^{25)}$ The DTaP-IPV-Hib-HepB vaccine can be administered for the primary series at the recommended ages or as a catch-up vaccination. In infants aged $\geq 7$ months, fewer than 3 doses are required for catch-up of the Hib component. If catch-up for other components is also needed in infants aged $\geq 7$ months, the DTaP-IPV-Hib-HepB vaccine can be used, but a 3-dose primary series should be completed to ensure an adequate immune response to those components. The DTaP-IPV-HibHepB vaccine should not be used for the booster dose when the 3 -dose primary series is completed. Any Hib conjugate vaccine licensed for use as a booster dose can be used. If DTaP-IPV-Hib$\mathrm{HepB}$ is inadvertently administered for the booster dose, it need not be repeated with another Hib-containing vaccine if the spacing between the previous doses was proper.

\section{Prevention of HepB}

Children are recommended to receive 3 doses of a HepB vaccine at birth and 1 and 6 months of age. ${ }^{26)}$ The DTaP-IPVHib-HepB vaccine is not licensed for the birth dose; rather, it is only indicated for the primary vaccination series in infants of HBsAg-negative mothers at ages 2, 4, and 6 months. In such cases, the HepB component is administered a total of 4 times at 0 , 2,4 , and 6 months of age (Table 3 ). If an infant is to be vaccinated with DTaP-IPV-Hib-HepB, the second dose of the HepB vaccine should not be administered at 1 month of age. Infants born to HBsAg-positive mothers are currently recommended to be immunized with HepB immunoglobulin at birth and then monovalent $\mathrm{HepB}$ vaccine at 0,1 , and 6 months. Although the Advisory Committee on Immunization Practices states that a hexavalent vaccine can be administered to infants at 2, 4, and 6 months of age regardless of maternal HBsAg status, ${ }^{27)}$ concerns persist about a longer interval between the first and second vaccine doses in countries with relatively higher endemicity, as most cases of $\mathrm{HepB}$ vaccine failure in high-risk newborns occur during the first few months of life. ${ }^{28,29)}$ According to a study in Thailand, after the control for maternal HBeAg status, when the interval between the first and second doses in newborns born to HBsAg-positive mothers exceeded 10 weeks, the infection risk of the newborn was 3.74 times higher (95\% CI, 0.97-14.39). ${ }^{30)}$
Administration of the second dose within 10 weeks of life did not increase the risk of infection. ${ }^{30)}$ However, since this was obtained using 4 or 5 doses of HepB vaccine without HepB immunoglobulin, careful consideration is needed to apply it to policy. making in Korea.

To ensure an adequate immune response, the last dose of the $\mathrm{HepB}$ vaccine should be administered at age $\geq 24$ weeks; therefore, administration of the third dose of DTaP-IPV-Hib$\mathrm{HepB}$ is not recommended before 24 weeks of age. If it is given earlier and other vaccine components than HepB are spaced properly, an additional dose of monovalent $\mathrm{HepB}$ vaccine should be administered at age $\geq 24$ weeks after the proper intervals between previous doses.

\section{Interchangeability}

There are limited data on the safety and immunogenicity of interchanging vaccines from different manufacturers in children. Whenever available, the same manufacturer's product should be used to complete the primary series. However, if the specific vaccine product previously administered is unavailable or unknown, the vaccination should not be deferred; rather, another manufacturer's product can be permitted. DTaP-IPV and DTaPIPV/Hib vaccines that contain similar acellular pertussis antigens from the same manufacturer may be used interchangeably if indicated for a patient's age.

\section{Minimum ages and intervals and simultaneous use of other vaccines}

The recommended minimum age for each dose and minimum intervals between doses for the administration of the DTaP-IPVHib-HepB vaccine are presented in Table 2. The DTaP-IPVHib-HepB vaccine can be administered simultaneously with other vaccines, such as the pneumococcal conjugate vaccine and rotavirus vaccine. When other vaccines are administered at the same visit, each should be injected at a separate site.

Table 3. Recommended immunization schedule for the prevention of diphtheria, tetanus, pertussis, poliomyelitis, invasive Hip and HepB using the tetravalent, pentavalent, or hexavalent vaccine

\begin{tabular}{|c|c|c|c|c|c|}
\hline Vaccine & At birth & 1 Month & 2 Months & 4 Months & 6 Months \\
\hline \multicolumn{6}{|c|}{ When vaccinated with tetravalent or pentavalent vaccine } \\
\hline Monovalent HepB & $\sqrt{a}^{\mathrm{a})}$ & $\sqrt{ }$ & & & $\sqrt{ }$ \\
\hline DTaP-IPV+Hib & & & $\sqrt{ } \sqrt{ }$ & $\sqrt{ } \sqrt{ }$ & $\sqrt{ } \sqrt{ }$ \\
\hline DTaP-IPV/Hib & & & $\sqrt{ }$ & $\sqrt{ }$ & $\sqrt{ }$ \\
\hline \multicolumn{6}{|c|}{ When vaccinated with hexavalent vaccine } \\
\hline Monovalent HepB & $\sqrt{ }$ & & & & \\
\hline DTaP-IPV-Hib-HepB & & & $\sqrt{ }$ & $\sqrt{ }$ & $\sqrt{ }$ \\
\hline
\end{tabular}

Hib, Haemophilus influenzae type b; HepB, hepatitis B; DTaP, diphtheria and tetanus toxoids and acellular pertussis; IPV, inactivated poliovirus.

a) The $\sqrt{ }$ represents the recommended vaccination period, while the number of $\sqrt{ }$ represents the number of injections. 


\section{Use of hexavalent vaccine in preterm infants}

The hexavalent vaccine can be administered to premature infants and should be vaccinated by age in the same schedule and manner as in term infants. Although a lower gestational age seems to be associated with lower antibody titers against some vaccine antigens (e.g., HepB, Hib, poliovirus type 1, and pertussis), the hexavalent vaccine showed good immunogenicity in preterm infants. ${ }^{31)}$ The hexavalent vaccine showed a similar or lower incidence of both local and systemic adverse events in preterm versus term infants, even when administered with other vaccines included in the routine immunization schedule. ${ }^{31)}$ No vaccinerelated severe adverse events were observed. ${ }^{31)}$ As reported by the European Medicines Agency, the potential risk of apnea and the need for respiratory monitoring for 48-72 hours should be considered when administering the primary series to very premature infants (born at $\leq 28$ weeks of gestation), particularly those with a previous history of respiratory immaturity. ${ }^{32)}$ As the benefit of vaccination is high in this group of infants, vaccinations should not be withheld or delayed.

\section{Contraindications and precautions}

The DTaP-IPV-Hib-HepB vaccine is contraindicated in infants with a history of a severe allergic reaction (e.g., anaphylaxis) after a previous dose or to a vaccine component. Vaccination in children with a serious allergic reaction (e.g., anaphylactic reaction) to any component of the vaccine is absolutely contraindicated. It should also not be administered to children who previously developed encephalopathy not attributable to another identifiable cause within 7 days of the administration of a previous dose of a pertussis component-containing vaccine. Vaccination should be delayed in children who have progressive or unstable neurologic disorders, uncontrolled seizures, or progressive encephalopathy until the neurologic status is clarified or the condition is stabilized. The vaccination should be chosen considering the risks and benefits in children who developed Guillain-Barré syndrome within 6 weeks of the administration of a previous dose of tetanus toxoid-containing vaccine.

\section{Footnotes}

Conflicts of interest: No potential conflict of interest relevant to this article was reported.

\section{References}

1. Halsey NA. Combination vaccines: defining and addressing current safety concerns. Clin Infect Dis 2001;33 Suppl 4:S312-8.

2. Skibinski DA, Baudner BC, Singh M, O'Hagan DT. Combination vaccines. J Glob Infect Dis 2011;3:63-72.

3. Dodd D. Benefits of combination vaccines: effective vaccination on a simplified schedule. Am J Manag Care 2003;9:S6-12.

4. Koslap-Petraco MB, Parsons T. Communicating the benefits of combination vaccines to parents and health care providers. J Pediatr Health Care 2003;17:53-7.

5. Lee SY, Hwang HS, Kim JH, Kim HH, Lee HS, Chung EH, et al. Immunogenicity and safety of a combined diphtheria, tetanus, acellular pertussis, and inactivated poliovirus vaccine (DTaP-IPV) compared to separate administration of standalone DTaP and IPV vaccines: a randomized, controlled study in infants in the Republic of Korea. Vaccine 2011;29:1551-7.

6. Kang JH, Lee HJ, Kim KH, Oh SH, Cha SH, Lee J, et al. The immunogenicity and safety of a combined DTaP-IPV//Hib vaccine compared with individual DTaP-IPV and Hib (PRP T) vaccines: a randomized clinical trial in South Korean infants. J Korean Med Sci 2016;31:1383-91.

7. Diphtheria, tetanus, pertussis (DTaP, Tdap, Td) vaccine. In: Kim JH, editor. Immunization guideline: 2018 report of the Committee on Infectious Diseases. 9th ed. Seoul (Korea): The Korean Pediatric Society, 2018:89-108.

8. Ministry of Food and Drug Safety. Full prescribing information-Hexaxim pre-filled syringe [Internet]. Cheongju (Korea): Ministry of Food and Drug Safety; c2020 [cited 2021 Apr 1]. Available from: https://nedrug. mfds.go.kr/pbp/CCBBB01/getItemDetail?itemSeq $=202002632$.

9. Obando-Pacheco P, Rivero-Calle I, Gómez-Rial J, Rodríguez-Tenreiro Sánchez C, Martinón-Torres F. New perspectives for hexavalent vaccines. Vaccine 2018;36:5485-94.

10. Syed YY. DTaP-IPV-HepB-Hib vaccine $\left(\operatorname{Hexyon}\left({ }^{\circledR}\right)\right)$ : an updated review of its use in primary and booster vaccination. Paediatr Drugs 2019;21:397-408.

11. Mukherjee P, Akpo EIH, Kuznetsova A, Knuf M, Silfverdal SA, Kosalaraksa P, et al. Hexavalent vaccines in infants: a systematic literature review and meta-analysis of the solicited local and systemic adverse reactions of two hexavalent vaccines. Expert Rev Vaccines 2021;20:31930.

12. Plotkin SA, Liese J, Madhi SA, Ortiz E. A DTaP-IPV//PRP T vaccine (Pentaxim): a review of 16 years' clinical experience. Expert Rev Vaccines 2011;10:981-1005.

13. World Health Organization. WHO - Pre-qualified vaccine [Internet]. Geneva (Switzerland): World Health Organization; c2021 [cited 2021 Apr 1]. Available from: https://extranet.who.int/pqweb/vaccines/prequalified-vaccines.

14. Kosalaraksa P, Chokephaibulkit K, Benjaponpitak S, Pancharoen C, Chuenkitmongkol S, B'Chir S, et al. Persistence of hepatitis B immune memory until 9-10 years of age following hepatitis $B$ vaccination at birth and DTaP-IPV-HB-PRP T vaccination at 2, 4 and 6 months. Hum Vaccin Immunother 2018;14:1257-65.

15. Vesikari T, Becker T, Vertruyen AF, Poschet K, Flores SA, Pagnoni MF, et al. A phase III randomized, double-blind, clinical trial of an investigational hexavalent vaccine given at two, three, four and twelve months. Pediatr Infect Dis J 2017;36:209-15.

16. Nunes MC, Madhi SA. Review of a new fully liquid, hexavalent vaccine: Hexaxim. Expert Opin Biol Ther 2013;13:575-93.

17. Aquino AG, Brito MG, Doniz CE, Herrera JF, Macias M, Zambrano B, et al. A fully liquid DTaP-IPV-Hep B-PRP-T hexavalent vaccine for primary and booster vaccination of healthy Mexican children. Vaccine 2012;30:6492-500.

18. López P, Arguedas Mohs A, Abdelnour Vásquez A, Consuelo-Miranda M, Feroldi E, Noriega F, et al. A randomized controlled study of a fully liquid DTaP-IPV-HB-PRP-T hexavalent vaccine for primary and booster vaccinations of healthy infants and toddlers in Latin America. Pediatr Infect Dis J 2017;36:e272-e82.

19. Kim YK, Vidor E, Kim HM, Shin SM, Lee KY, Cha SH, et al. Immunogenicity and safety of a fully liquid DTaP-IPV-HB-PRP T hexavalent vaccine compared with the standard of care in infants in the Republic of Korea. Vaccine 2017;35:4022-8.

20. Ceyhan M, Ylldırım İ, Tezer H, Devrim İ, Feroldi E. A fully liquid DTaPIPV-HB-PRP-T hexavalent vaccine for primary and booster vaccination of healthy Turkish infants and toddlers. Turk J Med Sci 2017;47:1247-56. 
21. European Medicines Agency. Scientific conclusions and grounds for the suspension of the marketing authorisation of Hexavac presented by the EMEA [Internet]. Amsterdam (Denmark): European Medicines Agency; c2005 [cited 2021 Mar 15]. Available from: https://www.ema.europa.eu/ en/documents/scientific-conclusion/hexavac-epar-scientific-conclusion_ en.pdf.

22. Jorgensen P, Poethko-Müller C, Hellenbrand W, Jilg W, Thierfelder W, Meyer C, et al. Low hepatitis B immunogenicity of a hexavalent vaccine widely used in Germany: results of the German Health Survey for Children and Adolescents, 2003-2006. Epidemiol Infect 2010;138:16219 .

23. Wenzel JJ, Jilg W. Loss of antibodies, but not of protection. Lancet Infect Dis 2010;10:738-9.

24. Poliovirus vaccine. In: Kim JH, editor. Immunization guideline: 2018 report of the Committee on Infectious Diseases. 9th ed. Seoul (Korea): The Korean Pediatric Society, 2018:109-17.

25. Haemophilus influenzae type b (Hib) vaccine. In: Kim JH, editor. Immunization guideline: 2018 report of the Committee on Infectious Diseases. 9th ed. Seoul (Korea): The Korean Pediatric Society, 2018:11831.

26. Hepatitis B vaccine. In: Kim JH, editor. Immunization guideline: 2018 report of the Committee on Infectious Diseases. 9th ed. Seoul (Korea): The Korean Pediatric Society, 2018:76-88.

27. Oliver SE, Moore KL. Licensure of a diphtheria and tetanus toxoids and acellular pertussis, inactivated poliovirus, Haemophilus influenzae type $b$ conjugate, and hepatitis b vaccine, and guidance for use in infants. Morb Mortal Wkly Rep 2020;69:136-9.

28. Poovorawan Y, Theamboonlers A, Vimolket T, Sinlaparatsamee S, Chaiear K, Siraprapasiri T, et al. Impact of hepatitis B immunisation as part of the EPI. Vaccine 2000;19:943-9.

29. Poovorawan Y, Sanpavat S, Pongpunlert W, Chumdermpadetsuk S,
Sentrakul P, Chitinand S, et al. Comparison of a recombinant DNA hepatitis $B$ vaccine alone or in combination with hepatitis B immune globulin for the prevention of perinatal acquisition of hepatitis $\mathrm{B}$ carriage. Vaccine 1990;8:S56-9; discussion S60-2.

30. Tharmaphornpilas P, Rasdjarmrearnsook A-o, Plianpanich S, Sanguanmoo P, Poovorawan Y. Increased risk of developing chronic HBV infection in infants born to chronically $\mathrm{HBV}$ infected mothers as a result of delayed second dose of hepatitis B vaccination. Vaccine 2009;27:6110-5.

31. Chiappini E, Petrolini C, Caffarelli C, Calvani M, Cardinale F, Duse M, et al. Hexavalent vaccines in preterm infants: an update by Italian Society of Pediatric Allergy and Immunology jointly with the Italian Society of Neonatology. Ital J Pediatr 2019;45:145.

32. European Medicines Agency. Hexaxim: Product information [Internet]. Amsterdam (Denmark): European Medicines Agency; c2020 [cited 2021 Apr 1]. Available from: https://www.ema.europa.eu/en/documents/ medicine-outside-eu/hexaxim-product-information_en.pdf.

33. World Health Organization. Public assessment summary reportHexaxim ${ }^{\circledR}$ [Internet]. Geneva (Switzerland): World Health Organization; c2015 [cited 2021 Mar 20]. Available from: https://www.who.int/ immunization_standards/vaccine_quality/pq_284_Hexaxim_1dose_SP_ Hexaxim_VPSAR.pdf.

How to cite this article: Cho HK, Park SE, Kim YJ, Jo DS, YK Kim, Eun BW, et al. Recommendation for use of diphtheria and tetanus toxoids and acellular pertussis, inactivated poliovirus, Haemophilus influenzae type $\mathrm{b}$ conjugate, and hepatitis $\mathrm{B}$ vaccine in infants. Clin Exp Pediatr 2021;64:602-7. https://doi. org/10.3345/cep.2021.00507 